\title{
Brain D-amino acids: a novel class of neuromodulators
}

\author{
Jean-Pierre Mothet $\cdot$ Solomon H. Snyder
}

Received: 6 September 2012 / Accepted: 7 September 2012

(c) Springer-Verlag 2012

In Alice in Through the Looking-Glass, and What Alice Found There, Alice proposes to her kitten a looking-glass house, whereupon Lewis Carroll fantasizes a backwards world and raises the question of life's chirality. Homochirality has been thought to be essential to biologygenerating distinct enzymes/receptors to cope with multiple isomers which would pose energetic burdens incompatible with life. Hence, we have D-sugars and L-amino acids. D-Amino acids found in mammalian tissues were assumed to be the by-products of dietary consumption, since plants and bacteria do synthesize D-amino acids. A nagging brain-teaser, for over 40 years, has been the existence of mammalian D-amino acid oxidase and D-aspartate oxidase with particularly high levels in the brain.

Like Alice, neurobiologists have now stepped through the looking-glass to characterize at least two D-amino acids, D-serine and D-aspartic acid, that may serve as the important signalling molecules in the nervous and endocrine systems. D-Aspartate was first found in the nervous system of marine mollusks and subsequently in nervous and endocrine tissues of many mammals, including humans. In 1992, Nishikawa and his colleagues developed a high performance liquid chromatography (HPLC) system

\section{J.-P. Mothet $(\bowtie)$}

Aix Marseille University, CRN2M UMR7286 CNRS,

51 Bd Pierre Dramard, 13344 Marseille Cedex 15, France

e-mail: jean-pierre.mothet@univ-amu.fr

\section{S. H. Snyder}

The Solomon H Snyder Department of Neuroscience,

Johns Hopkins University School of Medicine,

Baltimore, MD 21205, USA

e-mail: ssnyder@jhmi.edu to separate isomers in biological samples. They confirmed the presence of free D-aspartic acid in the brain of rodent, where they also identified free D-serine. Research by the authors and other groups identified the two enzymes, serine racemase and aspartate racemase, that synthesize $\mathrm{D}$-serine and D-aspartate, respectively. Emerging unanticipated roles for these two amino acids challenge fundamental dogma about cell-to-cell signaling. Thus, D-serine fulfils the criteria of a major, if not the sole, ligand for the strychnineinsensitive glycine modulatory binding site of the $N$-methyl-D-aspartate receptor (NMDA) subtype of glutamate receptor, which mediates excitatory neurotransmission and cognitive function. D-Aspartate mediates diverse neuroendocrine functions. We are just beginning to understand the importance of this novel class of brain messengers that will soon include additional candidates. Indeed, recent studies suggest that other D-amino acids, such as D-glutamate, $\mathrm{N}$-methyl-D-aspartate, D-alanine and D-glutamine, may also serve as messenger molecules. The expanded recognition of D-amino acids as signalling molecules, along with appreciation of gases as neuromodulators, further alters thinking about what should be a neurotransmitter.

This special volume of Amino Acids provides an overview of the current status of our understanding on D-amino acid biology, incorporating contributions from leaders in the field. We have assembled eight review articles that convey recent achievements characterizing the metabolism, brain disposition and roles in synaptic plasticity of D-amino acids, including the recent development of biosensors. The issue also incorporates two orignal studies by Prof Wang and his coworkers who report the contribution of spinal D-amino acid oxidase to bone cancer pain, and by Prof. Hamase and colleagues who demonstrate the alteration in $\mathrm{D}$-serine levels in mice lacking serine racemase and D-amino acid oxidase. 
The Guest Editors acknowledge all authors for their contributions as well as the reviewers for their personal dedication. The Guest Editors also express their gratitude to Professor Gert Lubec and to the publisher, SpringerVerlag, for the opportunity to prepare this special issue in Amino Acids. 